\title{
MUTATIONAL CONSERVATION, EVOLUTIONARY AND FUNCTIONAL UNDERSTANDING OF PROTO-ONCOGENE c-FOS
}

\section{Ruchi Yadav}

Amity Institute of Biotechnology, Amity University, Uttar Pradesh Lucknow 226028, UP, INDIA

Received - May 18, 2021; Revision - July 07, 2021; Accepted - August 16, 2021

Available Online-August 30, 2021

DOI: http://dx.doi.org/10.18006/2021.9(4).464.471

KEYWORDS
C-Fos
Proto-oncogene
Phylogenetic tree
UPGMA
MEGA

\begin{abstract}
c-Fos protein has a function in different types of cancers and is expressed mostly in neurons. It is a human homolog of the viral oncogene. c-Fos is a member of the FOS gene family, these genes interact with the JUN family member to form transcription factors and play a major role in neurons cell development. These genes were also used as an early marker, in neuronal cells to determine early growth and functional features of the neuroendocrine system. Losses in gene function due to mutation leads to neuronal death and have a function in apoptosis. This study has performed mutational conservation in the c-Fos gene across different species. the c-Fos protein sequence was retrieved from the UniProt database (P01100). Total forty nine (49) homologous sequences with the c-Fos protein sequence were identified using the BLASTp tool. Multiple sequence alignment (MSA) and phylogenetic tree construction was done using the MEGA tool. The phylogenetic tree shows that the c-Fos protein of Homosapiens was closely related to Pan troglodytes. UPGMA tree also shows the evolutionary relationship between c-Fos proteins and with the other 49 species included in the dataset. Evolutionary study shows that Myotis species was the common evolutionary species and predicted as root for all other species hence c-Fos gene might have an evolutionary link with these species. Myotis are the most wide diverged species and belongs to the genus of bats. This study highlights the similarity and evolutionary relationship of the c-Fos gene. In this research detailed analysis of evolutionary analysis, PPI, GO, Disease Enrichment was done to understand the functional and evolutionary aspects of c-FOS protein. This study identifies the evolutionary relationship, protein-protein interaction and pathway enrichment of the c-FOS protein. This research can be further extended to include ligand screening and identification of potential ligand against c-FOS protein for drug development and discovery.
\end{abstract}

* Corresponding author

E-mail: ryadav@1ko.amity.edu (Ruchi Yadav)

Peer review under responsibility of Journal of Experimental Biology and Agricultural Sciences.

Production and Hosting by Horizon Publisher India [HPI] (http://www.horizonpublisherindia.in/).

All rights reserved.
All the articles published by Journal of Experimental Biology and Agricultural Sciences are licensed under a Creative Commons Attribution-NonCommercial 4.0 International License Based on a work at www.jebas.org.

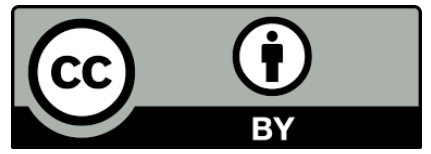




\section{Introduction}

The proto-oncogenes have been highly conserved over-evaluation as they play an important role in normal cellular function. These proto-oncogenes encode proteins that perform a normal cellular function in our body until any kind of mutation or alteration occurs, any kind of mutation leads to its conversion into an oncogene. These oncogenes are responsible for encoding some protein that causes cancer (Ding et al., 2020). The conversion of proto-oncogenes into oncogenes are either due to mutation or overexpression (Moench et al., 2019).

c-FOS is a gene that encodes protein FOS which is a protooncogene. FOS protein is a proto-oncogene associated with tumour formation and promotion. Researches in c-FOS protein have shown that it plays a significant role in several diseases like cancer especially cancer of the bone, and neurons etc. (Herrera \& Robertson, 1996). The FOS gene has been popularly studied in neuron related disorders (Sagata et al, 2017). Most of the proto oncogenes are homologs of viral oncogenes e.g. retroviral. Similarly, c-fos is also a human homolog of viral oncogenes (Zorzo et al., 2019; Ding et al., 2020). Its sequence relationship studies suggested that it is a viral analogue in the human genome. It is a small protein of 380 amino acids and found to be overexpressed in cancer cells. C-FOS belongs to the family of FOS transcription factors and has a leucine- Zipper motif that binds to DNA (Morandi-Raikova \& Mayer 2020).

The c Fos genes interact with the JUN family member to form transcription factor and play a major role in neurons cell development. JUN family members are proteins that dimerize with themselves and with c-Fos protein and have a function in DNA binding. Besides a role in neuron cells, it also regulates TGF beta which has an important role in signal transduction, cell proliferation and differentiation (Silva et al., 2019).

Mutation in genes is part of evolution and the underlying principle of evolution is genetic variation and its rise with successive mutation. Beneficial mutations in genes are selected by nature and are passed to next-generation (Stanisavljević et al, 2019). The c FOS is evolutionarily conserved which means that there are similar genes, portions of genes, or chromosome segments in different species which further reflect how different species has a common origin and this information plays an important functional property of the conserved element (Machado et al., 2018).

In the current era of genomics and proteomics that include high throughput technologies like Next generation Sequencing, Microarray technology, Mass spectrometry etc. along with the availability of sensitive bioinformatics software's and tools it has made it easier to understand the molecular and functional biology of genes and proteins (Waman et al., 2019). In this aspect bioinformatics tools and software's were used to highlights the important features of c-FOS protein that can be of high importance to signify its function in cancer. It is important to understand how a protein sequence of the c-FOS protein is conserved or related to other species Multiple Sequence Alignment (MSA) was performed, it produces an alignment table in which the pattern of amino acid conservation is represented and from this distant relationship can be detected.

It was also reported that most of the highly conserved protein sequences probably correspond to the active site. Building a phylogenetic tree requires multiple sequence alignment and the major goal of this work is to understand the molecular biology of the c-FOS protein. The objective behind phylogenetic tree construction is to predict the similarity of the c-FOS protein with other species oncogenes. These results also give detailed sequence conservation analysis like mutation, SNPs, Gene Conservation, Protein-Protein interaction and pathway enrichment of the c-FOS protein. This study also highlights the important functions of cFOS protein that can help researchers to identify potential ligands against this protein.

\section{Materials and Methods}

The protein sequence of the c-fos gene was retrieved from the UniProt database (https://www.uniprot.org/) with Uniprot id cFOS: P01100. Identification of homologous sequence was done using BLASTp (Protein Basic Local Alignment Search Tool) tool (https://blast.ncbi.nlm.nih.gov/) with default parameters. Figure 1 shows the workflow that was used for the construction of the Phylogenetic tree of c-FOS protein.

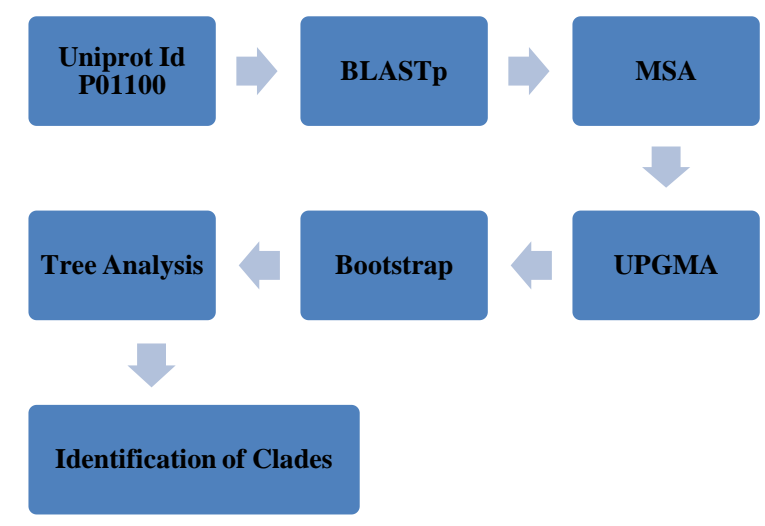

Figure 1 Methodology used for Phylogenetic tree construction

Identification of origin and relationship between various species has become an important field of research in understanding evolution. The proto-oncogene c-FOS encodes a normal protein, 
which is involved in cell proliferation and signal transduction. cFOS protein with UniProt id P01100 contains several motifs and domains that have been conserved during evolution. The Molecular Evolutionary Genetics Analysis (MEGA) tool ((https://www.megasoftware.net/) was used for the construction of a Phylogenetic tree by using the Unweighted pair group method with arithmetic mean (UPGMA) method (Santos et al., 2019). Several tools and software's are available for bioinformatic analysis of evolution and sequence conservation, in this research most widely used MEGA tool was used to identify the evolutionary link of c-FOS protein with other species.

Homologous sequence c-Fos was predicted using the BLASTp tool. Total 49 homologous protein sequences were identified that show similarity with c-FOS protein. Multiple sequence alignment (MSA) was done using CLUSTAL Omega (https://www.ebi.ac.uk/Tools/msa/clustalo/) to identify conserved regions. Further MSA result data was used for the construction of a phylogenetic tree using the MEGA tool. The phylogenetic tree was constructed for more understanding of evolutionary relationships using the UPGMA method, to identify closely related and neighbouring species. UPGMA tree also shows the evolutionary relationship between c-Fos proteins and with the other 49 species included in the dataset. Phylogenetic tree verification was done using the Bootstrap method with the value of 50 replicates using the MEGA tool and clades were identified.

\section{Results and Discussions}

To understand the conservation of amino acids, sequence motifs, domains MSA result was obtained from CLUSTAL Omega is visualized in MEGA Tool result of MSA is shown in figure 2. MSA result revealed that mutation at position 10: tyrosine residue was conserved across species. Further similarity tree was constructed using the UPGMA method, to identify closely related and neighbouring species.

\subsection{Evolutionary relationships of taxa}

The evolutionary history of c-FOS protein was obtained using the UPGMA method. The optimal tree was constructed with the branch length $=0.57100310$ (sum of branch length) as shown in Figure 3. The phylogenetic tree verification was done using the Bootstrap method (Lemoine et al., 2018). It is the method where replicates of MSA are generated by shuffling columns and trees are generated individually for each dataset. Finally, a consensus tree is constructed to obtain a bootstrap score. For this study total, 50 replicates were generated from the initial dataset and the bootstrap values are represented in branches as shown in figure 3 and 4 (Stott \& Bobay, 2020).

The consensus phylogenetic tree was made with the same parameters that were used for the construction of the initial phylogenetic tree by using the UPGMA method. The evolutionary distances between each species was calculated using the Poisson correction method (Lemoine et al., 2018), and this method includes the calculation of amino acids substitutions per site. c-FOS phylogenetic tree involves datasets including 49 amino acid sequences from different species. Errors in datasets like missing or some uncertain positions were deleted from each pair of sequences (pairwise deletion).

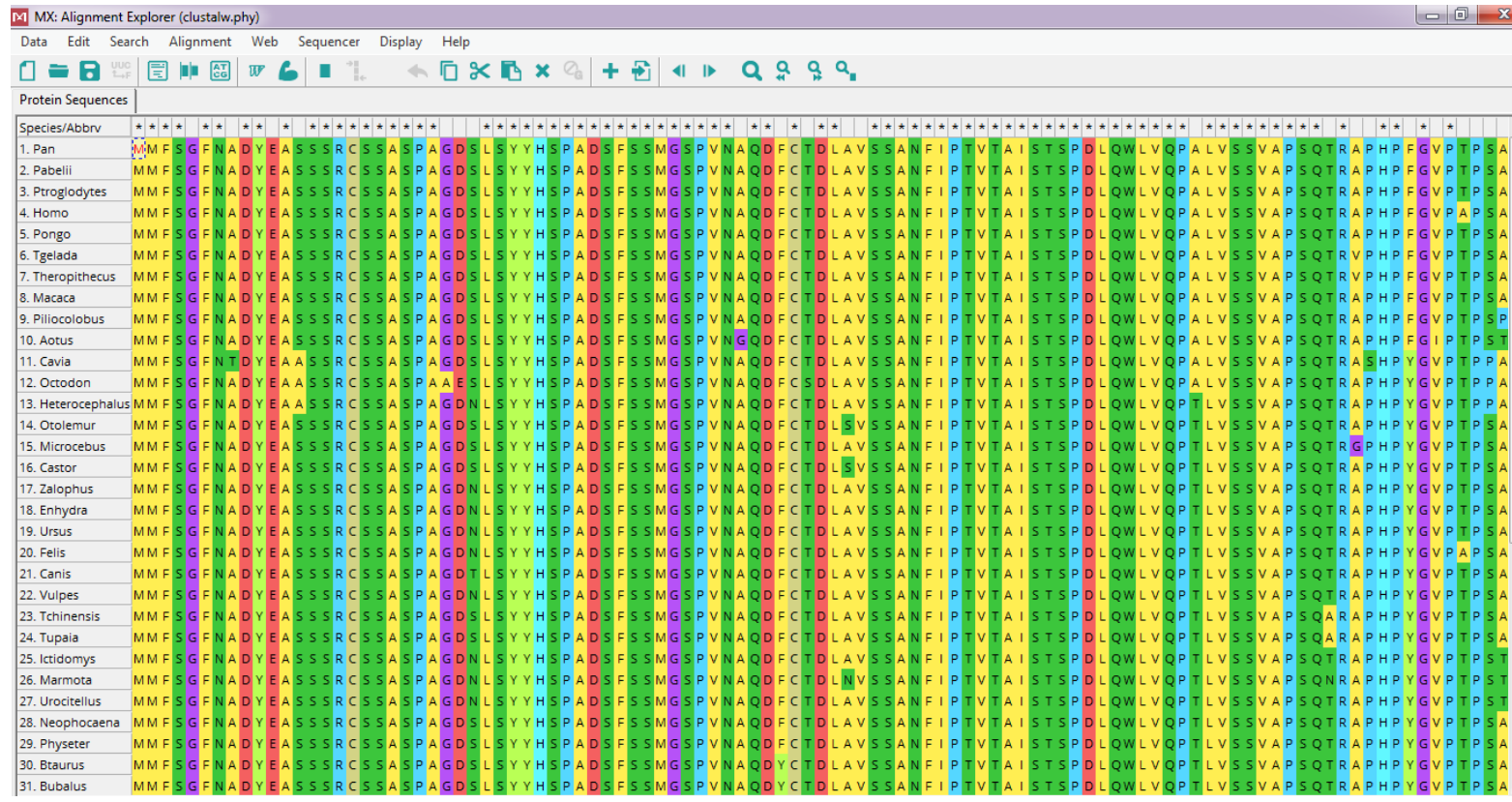

Figure 2 Result of Multiple sequence alignment of c-FOS protein using MEGA Tool

Journal of Experimental Biology and Agricultural Sciences http://www.jebas.org 


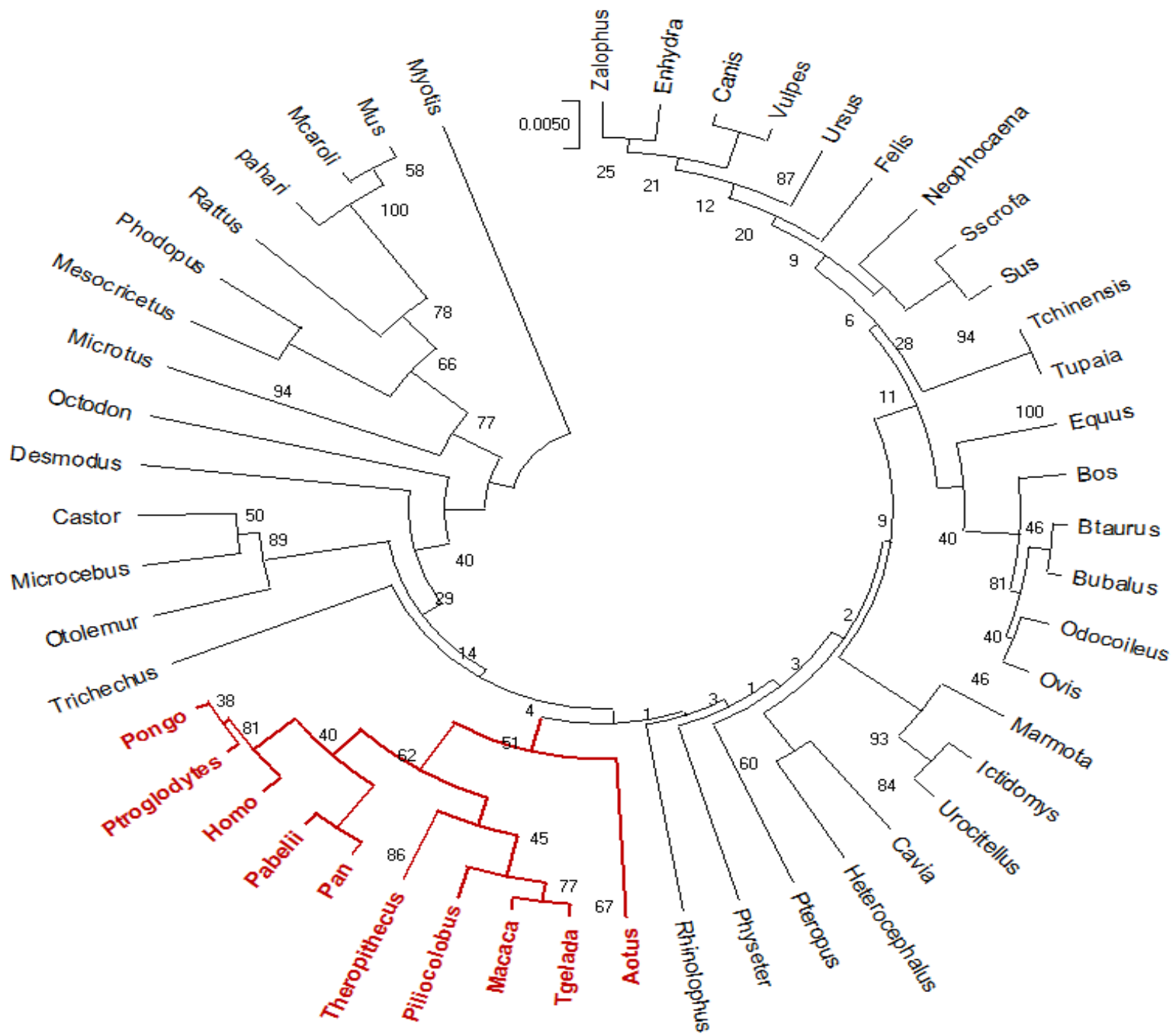

Figure 3 Phylogenetic tree of c-FOS protein constructed using UPGMA method and clade was identified (highlighted in red)

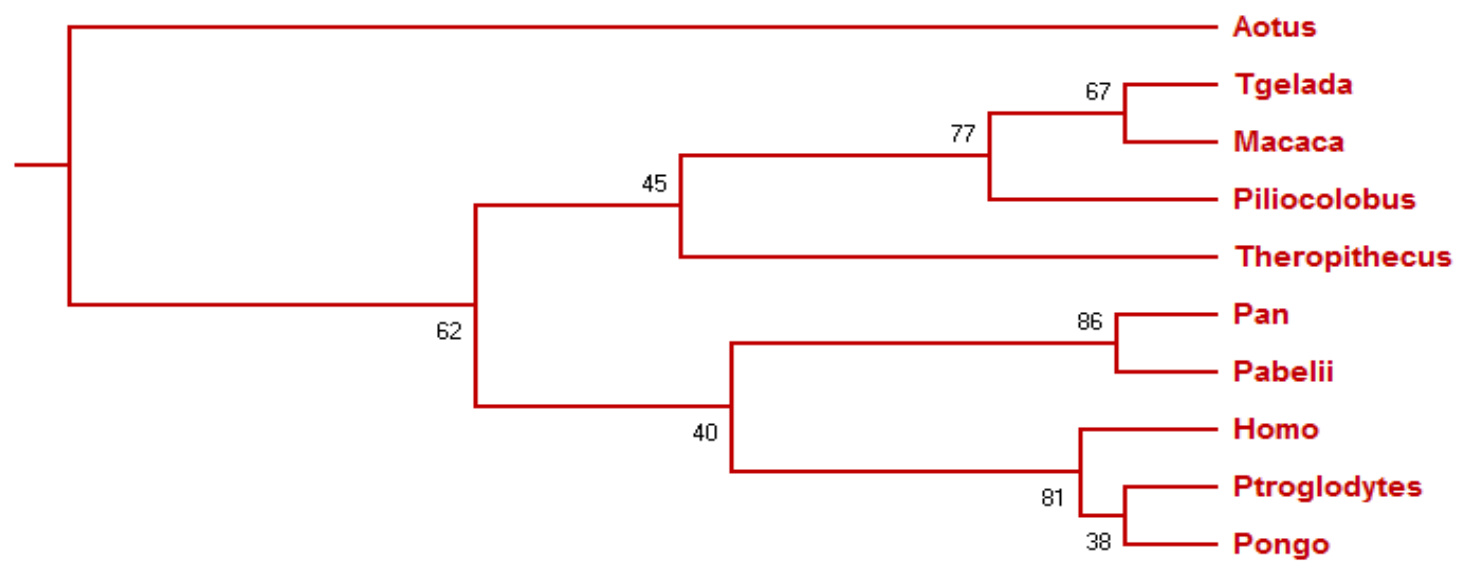

Figure 4 clade information extracted from Phylogenetic tree that shows closes neighbours along with bootstrap score

Journal of Experimental Biology and Agricultural Sciences http://www.jebas.org 


\subsection{Bootstrap Analysis of Phylogenetic Tree}

The bootstrap consensus tree was inferred from the datasets that include 50 replicates of initial MSA data (Zuckerkandl et al., 1965). This shuffled dataset of MSA was used for the construction of the evolutionary tree and for the calculation of the bootstrap score. Branches that correspond to a bootstrap value of less than $50 \%$ were deleted from the consensus tree since these branches do not depict any relationship with the species in the dataset. The percentage of replicates in the consensus tree that show values greater than $50 \%$ are represented in branches and shown in the tree for visualization of clades and neighbours and to identify closely related species (Kumar et al., 2018).

The phylogenetic tree shows that the c-Fos protein of Homosapiens was closely related to P. troglodytes. Evolutionary study shows that Myotis species was a common evolutionary root of all other species, hence c-Fos gene might have an evolutionary link with these species. Myotis are the most wide diverged species and belongs to the genus of bats. This study highlights the similarity and evolutionary relationship of the cFos gene.

\subsection{Protein-Protein Interaction Study}

The functional understanding of c-FOS protein work was extended to include protein-protein interaction (PPI) study, Gene Ontology (GO), and Pathway Enrichment. PPI analysis of c-FOS protein was done using the STRING database (https://string-db.org/2) as shown in Figure 5. PPI network was constructed with the following parameters: number of nodes (11), number of edges (34), average node degree (6.18), average local clustering coefficient (0.81), expected number of edges (10), PPI enrichment p-value (4.14e09 ). Clustering with the value of $k=3$ was done to identify closely related proteins as shown in figure 5 clusters are represented with different colours red, green, and blue.

PPI result shows that c-FOS protein interacts with 11 proteins viz: ATF2, CEBPA, ESR1, FOS, GATA3, HDAC1, JUN, JUNB, JUND, MAPK1 and STAT3. Functional annotation of all proteins is mentioned in table 1, which shows that all proteins are majorly involved in cell signalling pathways, cell development and signal transduction. Functional enrichment and Pathway analysis were done using Gene Ontology (GO) database to identify the process and function of the c-FOS protein (http://geneontology.org/).

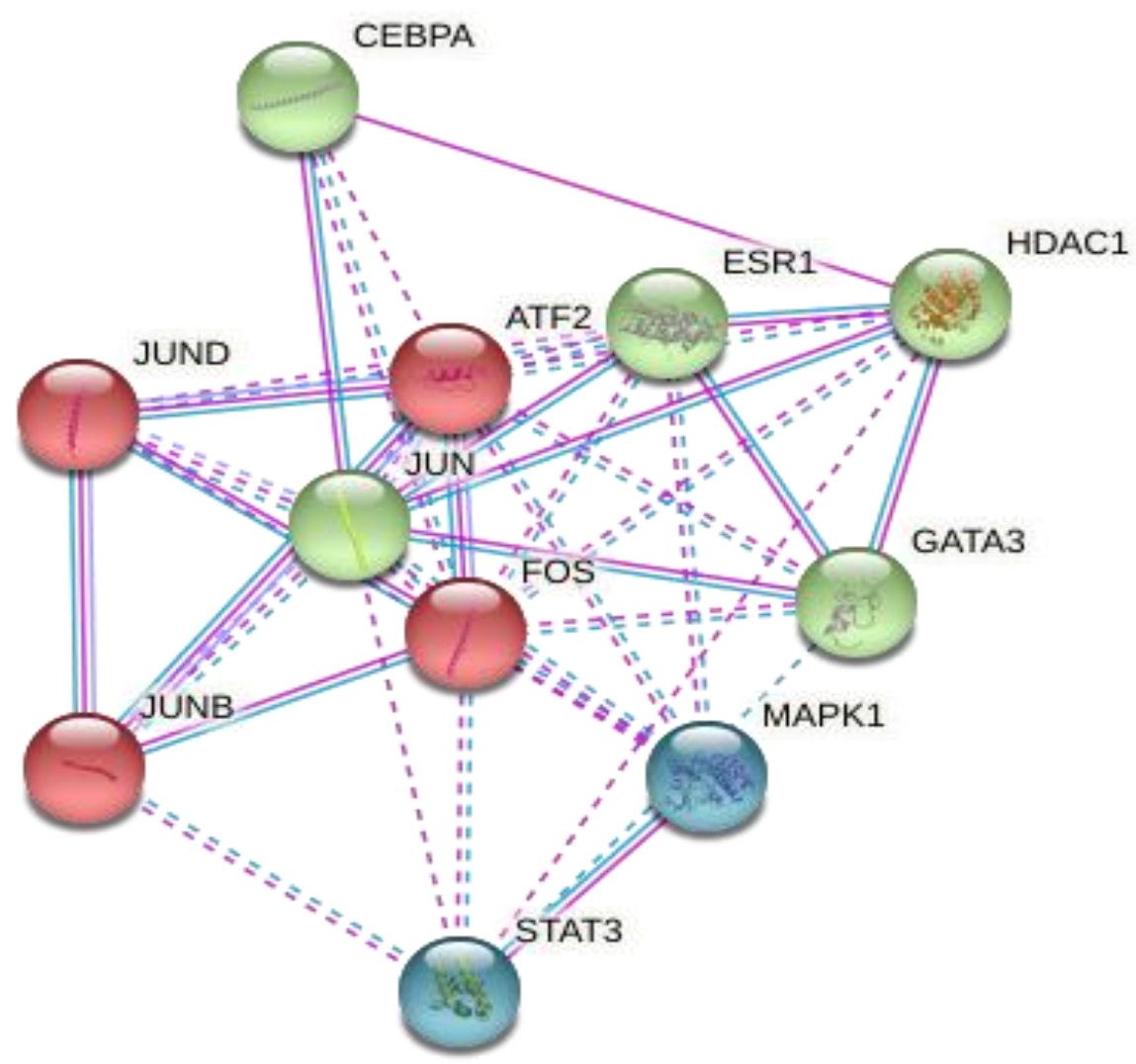

Figure 5 Protein-Protein interaction map of c-FOS protein and clustering with the value of $k=3$ (Cluster highlighted in red, green and blue) 
Table 1 List of proteins that shows interaction with c-FOS protein, predicted from PPI network through STRING Database

\section{S.No \#Node Annotation}

Cyclic AMP-dependent transcription factor ATF-2; Transcriptional activator which regulates the transcription of

1 ATF2 various genes, including those involved in anti-apoptosis, cell development, differentiation, and response to DNA damage and mutations (Li et al., 2019).

Transcription factor that coordinates proliferation arrest and the differentiation of myeloid progenitors, adipocytes,

2 CEBPA hepatocytes, and cells of the lung and the placenta. During early embryogenesis, play essential and redundant functions with CEBPB. It is an important protein for the development of the liver and lungs (Fatima et al., 2019).

\begin{tabular}{|c|c|c|}
\hline 3 & ESR1 & $\begin{array}{l}\text { Estrogen receptor also called as Nuclear hormone receptor. ESR1 is associated with the gene regulation process like } \\
\text { eukaryotic gene expression, gene interaction, the expression also it has a function in cell differentiation, development } \\
\text { and proliferation (Gu et al., 2021). }\end{array}$ \\
\hline 4 & FOS & $\begin{array}{l}\text { It is a Proto-oncogene that has homologs in the viral genome also. It is a DNA binding protein having Leucine Zipper } \\
\text { motif it binds to DNA. It interacts with transcription factor proteins like JUN protein and its family members also. This } \\
\text { protein is associated with the formation of cell development and differentiation. FOS protein has a function in } \\
\text { signalling pathways and signals transduction pathways (Stanisavljević et al., 2019). }\end{array}$ \\
\hline 5 & GATA3 & $\begin{array}{l}\text { Trans-acting T-cell-specific transcription factor GATA-3. Binds to the consensus sequence 5'-AGATAG-3'. Required } \\
\text { for the T-helper } 2 \text { (Th2) differentiation process following immune and inflammatory responses (Almutairi et al., 2019). }\end{array}$ \\
\hline 6 & HDAC1 & $\begin{array}{l}\text { Histone deacetylase } 1 \text { is an important protein that has a function in transcriptional regulation at different levels of the } \\
\text { cell cycle, development and differentiation. It is involved in the deacetylation of core histones ( } \mathrm{H} 2 \mathrm{~A}, \mathrm{H} 2 \mathrm{~B}, \mathrm{H} 3 \text { and } \mathrm{H} 4) \\
\text { at the lysine amino acids that are responsible for the epigenetic regulation of gene expression (Wu et al., 2020) }\end{array}$ \\
\hline 7 & JUN & $\begin{array}{l}\text { Transcription factor AP-1 has a function in the signalling pathway and is also involved in stimulation. Binds to the } \\
\text { USP } 28 \text { promoter in colorectal cancer (CRC) cells; JUN proteins have a basic leucine zipper domain (Nguyen et al., } \\
\text { 2021). }\end{array}$ \\
\hline 8 & JUNB & $\begin{array}{l}\text { Transcription factor JUN-B; these transcription factors are involved in regulating gene function and regulate the } \\
\text { expression of growth factors (Kallergi et al., 2019). }\end{array}$ \\
\hline 9 & JUND & $\begin{array}{l}\text { Transcription factor JUN-D; these transcription factor binding AP-1 sites; Belongs to the bZIP family. JUND proteins } \\
\text { belong to the JUN subfamily (Singh et al., 2020) }\end{array}$ \\
\hline 10 & MAPK1 & $\begin{array}{l}\text { Mitogen-activated protein kinase } 1 \text { is an important protein that is most widely studied to understand the molecular } \\
\text { mechanism of cell signalling and signal transduction. It is a kinase protein that has an essential role in the MAP } \\
\text { pathway. It is also associated with a cascade of enzymatic reactions activated by MAP/ERK pathways. The function of } \\
\text { this protein is associated with cell growth, development, differentiation, regulation survival, translation and } \\
\text { cytoskeletal rearrangements (Zhu et al., 2020). }\end{array}$ \\
\hline 11 & STAT3 & $\begin{array}{l}\text { STAT (Signal transducer and activator of transcription 3) is a Signal transducer protein that acts as an initiator of the } \\
\text { transcription process and facilitates cellular responses to interleukins, KITLG/SCF, LEP and other growth factors. } \\
\text { Activated by IL31 through IL31RA. Acts as a regulator of the inflammatory response by regulating (Shui et al., 2019). }\end{array}$ \\
\hline
\end{tabular}

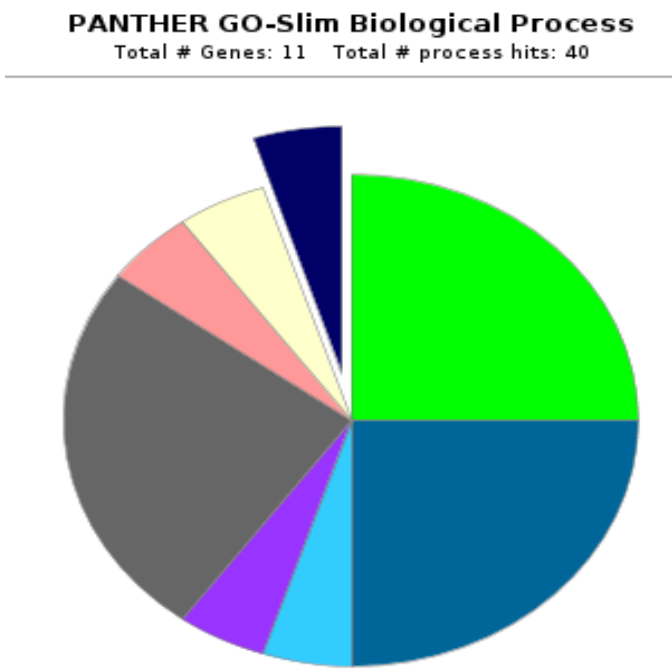

Biological regulation (GO:0065007)

Cellular process (GO:0009987)

Developmental process (GO:0032502)

Immune system process (GO:0002376)

Metabolic process (GO:0008152)

Multicellular organismal process (GO:0032501)

Response to a stimulus (GO:0050896)

Signalling (GO:0023052)

Figure 6 GO result of 11 proteins shows that c-FOS protein has a function in biological regulation and others processes as shown above. Form predicted GO signalling is highlighted with the GO:0023052 and highlighted in the chart

Journal of Experimental Biology and Agricultural Sciences http://www.jebas.org 


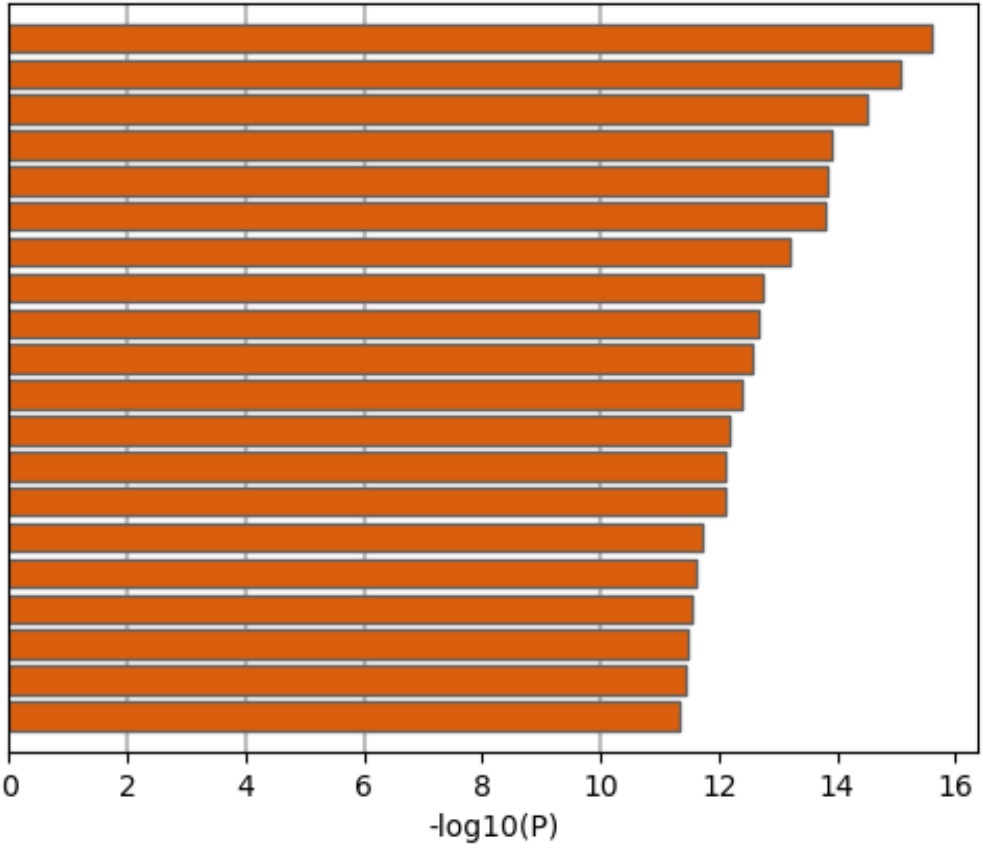

\author{
Tumor Promotion \\ Myeloid Leukemia \\ Adult Classical Hodgkin Lymphoma \\ Polycystic Kidney Diseases \\ Polycystic Kidney, Autosomal Dominan \\ Classical Hodgkin's Lymphoma \\ Rainbow Trout Hepatoma \\ androgen independent prostate cancel \\ Skin Carcinogenesis \\ Amyloidosis cutis dyschromia \\ Zinc deficiency \\ Immune System Diseases \\ Cytomegalovirus Infections \\ Anemia of chronic disease \\ Avellino corneal dystrophy \\ Perihilar Cholangiocarcinoma \\ Adult T-Cell Lymphoma/Leukemia \\ Penile hypospadias \\ Lupus Erythematosus \\ Ki-1+ Anaplastic Large Cell Lymphoma
}

Figure 7 Functional Enrichment of all 11 proteins using DisGeNET database

3.4 Gene Ontology (GO) and Pathway Enrichment of Network

GO analysis of all 11 proteins as mentioned in table 1 was used for GO analysis (Khanal \& Patil, 2020). These proteins were also used for process and pathway enrichment as shown in figure 6 .

GO analysis shows that interacted proteins are associated with signalling with the GO id: GO:0023052, biological regulation (GO:0065007), developmental process (GO:0032502), metabolic process (GO:0008152) etc.

Enrichment analysis of all 11 proteins as mentioned earlier in table 1 was also done with the DisGeNET Database (Disease Association Database Of Gene-Disease Associations) (https://www.disgenet.org/). Functional enrichment association with disease database is shown in figure 7 . Results of the study revealed that most of the proteins that interact with c-FOS protein are associated with tumour formation with a Tumor Promotion count value of 8 percentage, GO C1519689, 73\% of total protein, $16.00 \log 10(\mathrm{P})$ and $-11.00 \log 10(\mathrm{q})$ values.

\section{Conclusion}

The c-Fos protein of Homosapiens was closely related to $P$. troglodytes. $P$. troglodytes is commonly known as chimpanzees which is a species of great apes found mainly in Africa. Other than this Myotis species was a common evolutionary root of all other species, hence the c-Fos gene might have an evolutionary link with these species. Myotis are the most wide diverged species and belongs to the genus of bats. The similarity of the c-FOS protein was observed with 49 species, these results highlighted the diversity of species and how c-FOS is conserved among them. PPI result shows that c-FOS protein interacts with 11 proteins viz: ATF2, CEBPA, ESR1, FOS, GATA3, HDAC1, JUN, JUNB, JUND, MAPK1 and STAT3 and these proteins are functional in signal transduction and signalling pathway. Further GO and Disease enrichment analysis was also done for a deeper understanding of c-FOS protein and the results of the study also revealed that interacted proteins are associated with the tumour formation. Detailed results of GO, Functional enrichment established the importance of c-FOS protein in the tumour formation.

\section{Conflict of Interest - Nil}

\section{References}

Almutairi B, Charlet J, Dallosso AR, Szemes M, Etchevers HC, Malik KT, Brown KW (2019) Epigenetic deregulation of GATA3 in neuroblastoma is associated with increased GATA3 protein expression and with poor outcomes. Scientific Reports 9(1):1-12.

Ding Y, Hao K, Li Z, Ma R, Zhou Y, Zhou Z, et al. (2020) c-Fos separation from Lamin A/C by GDF15 promotes colon cancer invasion and metastasis in inflammatory microenvironment. Journal of Cellular Physiology 235(5):4407-4421.

Fatima LA, Campello RS, Barreto-Andrade JN, Passarelli M, Santos RS, Clegg DJ, Machado UF (2019) Estradiol stimulates adipogenesis and Slc2a4/GLUT4 expression via ESR1-mediated activation of CEBPA. Molecular and Cellular Endocrinology 498:110447. 
Gu G, Tian L, Herzog SK, Rechoum Y, Gelsomino L, Gao M, et al. (2021) Hormonal modulation of ESR1 mutant metastasis. Oncogene 40(5):997-1011.

Herrera DG, Robertson HA (1996) Activation of c-fos in the brain. Progress in Neurobiology 50(2-3):83-107. https://doi.org/ 10.1016/ s0301-0082(96)00021-4

Kallergi G, Tsintari V, Sfakianakis S, Bei E, Lagoudaki E, Koutsopoulos A, et al. (2019) The prognostic value of JUNBpositive CTCs in metastatic breast cancer: from bioinformatics to phenotypic characterization. Breast Cancer Research 21(1):1-13.

Khanal P, Patil BM (2020) Gene ontology enrichment analysis of $\alpha$-amylase inhibitors from Duranta repens in diabetes mellitus. Journal of Diabetes \& Metabolic Disorders 19:735-47.

Kumar S, Stecher G, Li M, Knyaz C, Tamura K (2018) MEGA X: Molecular Evolutionary Genetics Analysis across computing platforms. Molecular Biology and Evolution 35:1547-1549.

Lemoine F, Entfellner JBD, Wilkinson E, Correia D, Felipe MD, De Oliveira T, Gascuel O (2018) Renewing Felsenstein's phylogenetic bootstrap in the era of big data. Nature 556(7702):452-456.

Li M, Zhang D, Ge X, Zhu X, Zhou Y, Zhang Y, et al. (2019) TRAF6-p38/JNK-ATF2 axis promotes microglial inflammatory activation. Experimental Cell Research 376(2): 133-148.

Machado NL, Abbott SB, Resch JM, Zhu L, Arrigoni E, Lowell BB, et al. (2018) A glutamatergic hypothalamomedullary circuit mediates thermogenesis, but not heat conservation, during stressinduced hyperthermia. Current Biology 28(14): 2291-2301.

Moench KM, Breach MR, Wellman CL (2019) Chronic stress produces enduring sex-and region-specific alterations in novel stressinduced c-Fos expression. Neurobiology of Stress 10: 100147.

Morandi-Raikova A, Mayer U (2020) The effect of monocular occlusion on hippocampal c-Fos expression in domestic chicks (Gallus gallus). Scientific Reports 10(1): 1-13.

Nguyen NT, Du K, Akakpo JY, Umbaugh DS, Jaeschke H, Ramachandran A (2021) Mitochondrial protein adduct and superoxide generation are prerequisites for early activation of c-jun $\mathrm{N}$-terminal kinase within the cytosol after an acetaminophen overdose in mice. Toxicology Letters 338: 21-31.

Sagata N, Kato TA, Kano SI, Ohgidani M, Shimokawa N, SatoKasai M, et al. (2017) Dysregulated gene expressions of MEX3D, FOS and BCL2 in human induced-neuronal (iN) cells from NF1 patients: a pilot study. Scientific Reports 7(1): 1-8.
Santos IGD, Carneiro VQ, Silva Junior ACD, Cruz CD, Soares PC (2019) Self-organizing maps in the study of genetic diversity among irrigated rice genotypes. Acta Scientiarum Agronomy 41:DOI: https://doi.org/10.4025/actasciagron.v41i1.39803.

Shui X, Chen S, Lin J, Kong J, Zhou C, Wu J (2019) Knockdown of lncRNA NEAT1 inhibits Th17/CD4+ T cell differentiation through reducing the STAT3 protein level. Journal of Cellular Physiology 234(12): 22477-22484.

Silva BA, Burns AM, Gräff J (2019) A cFos activation map of remote fear memory attenuation. Psychopharmacology 236(1): 369-381.

Singh G, Fritz SE, Seufzer B, Boris-Lawrie K (2020) The mRNA encoding the JUND tumor suppressor detains nuclear RNAbinding proteins to assemble polysomes that are unaffected by mTOR. Journal of Biological Chemistry 295(22): 7763-7773.

Stanisavljević A, Perić I, Gass P, Inta D, Lang UE, Borgwardt S, Filipović D (2019) Brain sub/region-specific effects of olanzapine on c-Fos expression of chronically socially isolated rats. Neuroscience 396: 46-65.

Stott CM, Bobay LM (2020) Impact of homologous recombination on core genome phylogenies. BMC genomics 21(1): 1-10.

Waman VP, Vedithi SC, Thomas SE, Bannerman BP, Munir A, Skwark MJ, et al. (2019) Mycobacterial genomics and structural bioinformatics: opportunities and challenges in drug discovery. Emerging Microbes \& Infections 8(1): 109-118.

Wu CC, Jin LW, Wang IF, Wei WY, Ho PC, Liu YC, Tsai KJ (2020) HDAC1 dysregulation induces aberrant cell cycle and DNA damage in progress of TDP-43 proteinopathies. EMBO Molecular Medicine 12(6): e10622.

Zhu S, Song W, Sun Y, Zhou Y, Kong F (2020) MiR-342 attenuates lipopolysaccharide-induced acute lung injury via inhibiting MAPK1 expression. Clinical and Experimental Pharmacology and Physiology 47(8): 1448-1454.

Zorzo C, Méndez-López M, Méndez M, Arias JL (2019) Adult social isolation leads to anxiety and spatial memory impairment: Brain activity pattern of COx and c-Fos. Behavioural Brain Research 365: 170-177.

Zuckerkandl E, Pauling L (1965) Evolutionary divergence and convergence in proteins. In: Bryson V, Vogel HJ (Eds.) Evolving Genes and Proteins, Academic Press, New York, Pp. 97-166. 